Pathologe 2017 · 38:278-285

DOI 10.1007/s00292-017-0312-y

Online publiziert: 22. Juni 2017

(c) Der/die Autor(en) 2017. Dieser Artikel ist

eine Open-Access-Publikation.

Schwerpunktherausgeberin

A. M. Müller, Bonn

CrossMark

\section{Hintergrund}

Maligne solide Tumoren des Kindesalters umfassen morphologisch ein extrem breites Spektrum, und auch die häufigsten soliden Tumoren der Kindheit sind seltene Entitäten. Pro Jahr erkrankt ungefähr eines von 100.000 Kindern unter 15 Jahren an einem Neuroblastom, welches der häufigste extrakranielle solide Tumor des Kindesalters ist. Rhabdoidtumoren treten mit einer Häufigkeit von 1,5 Fällen/Jahr unter 10.000.000 Kindern unter 15 Jahren auf. Daher wird der überwiegende Anteil aller Tumoren in europaweite Protokolle eingebracht, um ausreichend Daten über Verlauf, Prognose, Therapiewirksamkeit und mögliche neue therapeutische Optionen sammeln zu können.

In den letzten Jahrzehnten hat sich dank dieser Studien immer deutlicher herauskristallisiert, dass jene Tumoren, die typischerweise während des Kindesalters auftreten, oft eine völlig unterschiedliche histogenetische Entstehung im Vergleich zu Tumoren des Erwachsenenalters aufweisen.

Die Genetik spielt bei der korrekten Diagnose und dem weiteren therapeutischen Prozedere eine immer wichtigere Rolle. Üblicherweise sind in jenen Zentren, in denen Kindertumoren operiert, diagnostiziert und weiterfolgend therapiert werden, für die pathologische Aufarbeitung bereits Leitlinien (sogenannte Standing Operating Procedures [SOPs]) etabliert, die die notwendige qualitativ hochwertige Aufarbeitung der Tumoren gewährleisten. Diese Leitlinien berücksichtigen nicht nur die örtlichen Gegebenheiten, sondern ins-

\author{
B. Gürtl-Lackner ${ }^{1,2} \cdot$ D. Gisselsson-Nord' · G. Vujanic ${ }^{3}$ \\ ${ }^{1}$ Institut für Pathologie, Labmedicin Skåne, Lund, Schweden \\ ${ }^{2}$ Institut für Pathologie, Medizinische Universität Graz, Graz, Österreich \\ ${ }^{3}$ Abteilung für Zelluläre Pathologie, Universitätsklinikum Wales, Universität Cardiff, Cardiff, Großbritannien
}

\title{
Solide Kindertumoren
}

\section{Ein Streifzug durch das Raritätenkabinett}

besondere die einzelnen internationalen Protokolle. Zur Gewährleistung einer qualitativ gleichwertigen Beurteilung im Rahmen des Studienprotokolls muss Material oftmals für histologische und/ oder genetische Untersuchungen an zentrale SIOP-autorisierte (International Society of Paediatric Oncology) Reviewboards, wie beispielsweise das Deutsche Kindertumorregister in Kiel oder die entsprechenden nationalen Reviewboards der einzelnen europäischen Länder, versandt werden. Solide Kindertumoren sollen immer unfixiert an eine mit den Leitlinien vertraute Pathologie versandt und dann entsprechend den Vorgaben verarbeitet werden.

Der folgende Artikel gibt einen Einblick in die spezielle Diagnostik solider Kindertumoren. Dafür haben wir $3 \mathrm{Tu}-$ morentitäten ausgewählt, die nicht auf ein Organ beschränkt auftreten und im Erwachsenenalter lediglich in Einzelfällen in der Literatur beschrieben werden.

\section{Neuroblastom}

Neuroblastome gehören zu den häufigsten extrakraniellen soliden Tumoren der Kindheit. Die Tumoren entstehen aus Gewebe des sympathischen Nervensystems wie beispielsweise der Nebenniere [15].

\section{Makroskopische Beurteilung}

Eine korrekte makroskopische Aufarbeitung ist für die Qualität der Diagnosestellung essenziell, daher wurde dies in einheitlichen europäischen Richtlinien festgelegt. Dabei müssen von mindestens 2 unterschiedlichen Tumorarealen zytologische Abklatschpräparate (Tupf- präparate) angefertigt und dazu korrespondierende Tumoranteile eingefroren werden. Mithilfe eines Gefrier- oder Paraffinschnitts muss überprüft werden, $\mathrm{ob}$ ein minimaler Gehalt von $60 \%$ Tumorzellen für genetische Analysen und $20 \%$ für Ploidieanalysen in den Proben enthalten ist (-Infobox 1). Genetische Untersuchungen von Ganglioneuroblastomen und -neuromen können nur unter speziellen Bedingungen durchgeführt werden, um eine Unterscheidung zwischen Tumorzellen und Schwannzellen zu ermöglichen (beispielsweise Mikrodissektion). Die genetischen Untersuchungen der Tumoren werden in zentralen Referenzlaboren durchgeführt [1].

Ein wichtiges Kriterium für einen möglichen aggressiven klinischen Verlauf von Neuroblastomen ist die Metastasierung ins Knochenmark. In den Konsensuskriterien wird daher die quantitative Bewertung von bilateralen Knochenmarkstanzen und von Knochenmarkaspirat festgelegt [8].

\footnotetext{
Infobox 1 Neuroblastom

- Standard: Tumortupfpräparate in Alkohol und bei RT fixieren. Wichtig: Farbmarkierung der Resektionsränder erst nach Tupfpräparatabnahme und Entnahme der Proben für die Genetik.

- Für genetische Untersuchungen unfixiertes Gewebe aus 2 Tumorarealen asservieren.

- Makroskopische Beurteilung und Dokumentation (ggf. Foto) aller Herde/ separater Knoten erforderlich, da eine spätere/nachträgliche histologische Rekonstruktion oftmals unmöglich ist.
} 


\section{Tab. 1 Histologische Begutachtung von Neuroblastomen}

\begin{tabular}{|c|c|c|c|}
\hline Parameter & Hoch & Mittel & Gering \\
\hline$M K I$ & $>4 \%$ & $2-4 \%$ & $<2 \%$ \\
\hline Zellularität & $\begin{array}{l}\text { 700-900 Neuroblas- } \\
\text { ten/,high power field“ } \\
\text { (HPF) }\end{array}$ & $\begin{array}{l}400-600 \text { Neuroblas- } \\
\text { ten/HPF }\end{array}$ & $\begin{array}{l}100-300 \text { Neuroblas- } \\
\text { ten/HPF }\end{array}$ \\
\hline
\end{tabular}

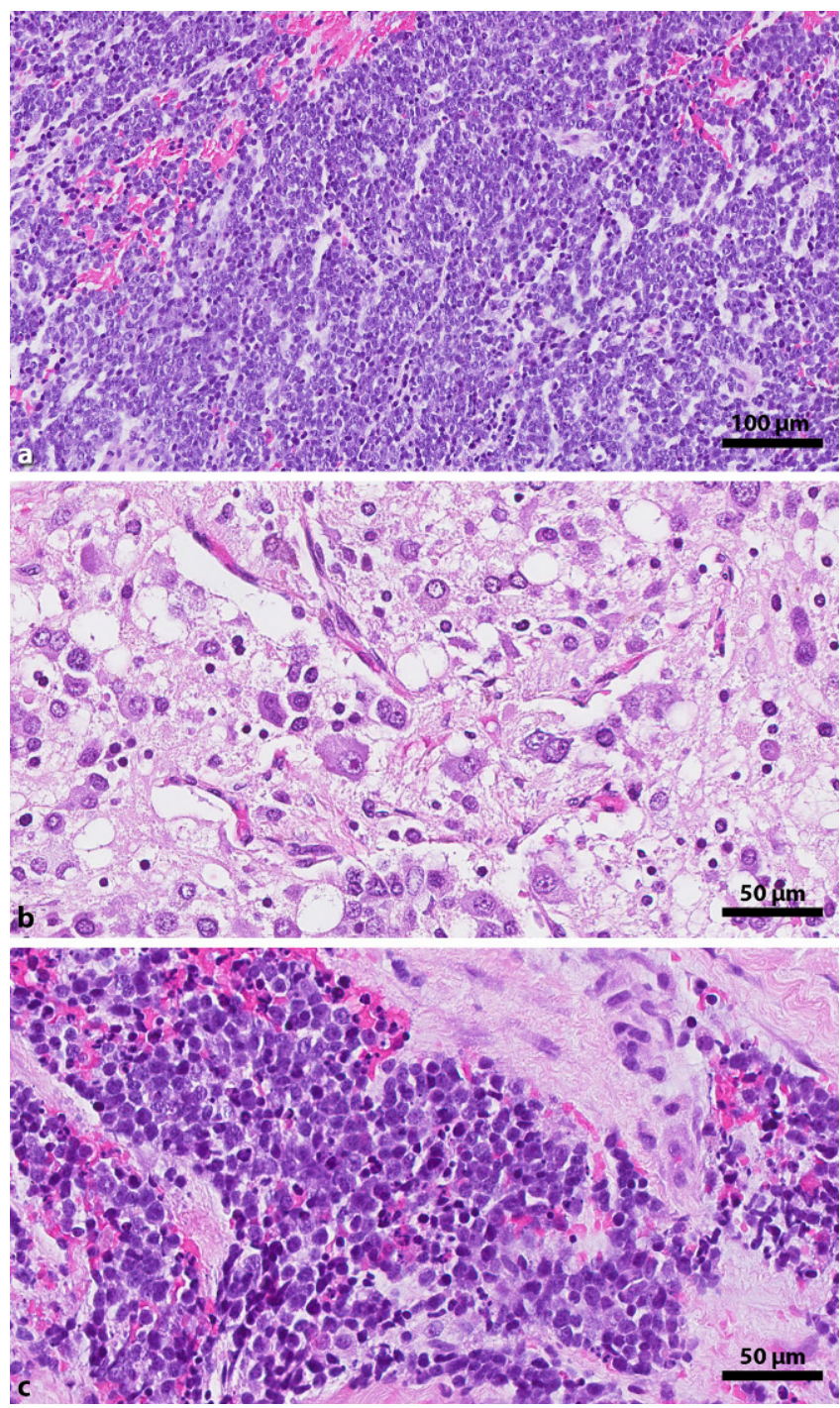

Abb. $1<$ a Unreifes Neuroblastom, morphologisch einem klein-rund-blauzelligen Tumor entsprechend. b Differenzierende Neuroblasten mit synchroner Ausreifung von Zytoplasma und Kern. c Mitose- und Karyorhexisfiguren in einem Neuroblastom; eine semiquantitative Beurteilung ist Bestandteil der histopathologischen Beurteilung

\section{Histologie und histologische Subtypen}

Histologisch bestehen Neuroblastome aus unreifem neuronalem Gewebe. Die histologische Klassifikation erfolgt anhand der International Neuroblastoma Pathology Classification (INPC). Grundsätzlich wird dabei zwischen schwannzellstromareichen/schwannzellstromaprädominanten Tumoren, nämlich Ganglioneuromen und -neuroblastomen, und schwannzellstromaarmen Tumoren, den Neuroblastomen im eigentlichen Sinn, unterschieden.

Neuroblastome werden anhand des Differenzierungsmusters der unreifen Neuroblasten in undifferenzierte, wenig differenzierte und differenzierende Neuroblastome unterteilt.

\section{MKI}

Diehistologische Begutachtung von Neuroblastomen muss auch die Beurteilung des Mitose-Karyorhexis-Index (MKI) enthalten (•Tab. 1). In die Beurteilung werden 5000 unreife Neuroblasten einbezogen, abhängig von der Zellularität müssen entsprechend viele $\mathrm{HPFs}$ („high power fields“) ausgezählt werden. Hohe Zellularität ist dabei definiert mit 700-900 Neuroblasten/HPF, mittlere Zellularität mit 400-600 Zellen/HPF und niedrige Zellularität mit $100-300$ Zellen/HPF. Der MKI wird ebenfalls anhand eines dreiteiligen Schemas unterteilt: geringer MKI $<2 \%$, mittlerer MKI 2-4\%, hoher MKI > $4 \%$ (•Abb. 1c). Die Mitoserate wird mit mehr oder weniger als 10 Mitosen/10 HPF gewertet.

Kalzifikationen müssen auch dokumentiert werden. Die Parameter MKI, Mitoserate und Kalzifikation gehen in die weitere Prognose ein.

\section{Undifferenzierte Neuroblastome}

Für die eindeutige Diagnose des undifferenzierten Neuroblastoms ( $\mathbf{0}$ Abb. 1a) sind weitere immunhistochemische Untersuchungen mit Antikörpern gegen Chromogranin, Synaptophysin und Neuroblastommarker (NB84) zur Abgrenzung gegenüber anderen kleinen rundzelligen Tumoren wie beispielsweise Rhabdomyosarkome oder Lymphome notwendig $[21,25,26]$. Einen wichtigen negativen prognostischen Faktor der gering differenzierten Neuroblastome stellen prominente, eosinophile Nukleolen („,bull's eyes") in bereits etwas größeren blasigen Zellkernen dar [29].

\section{Wenig differenzierte Neuroblastome}

In wenig differenzierten Neuroblastomen findet man zumindest stellenweise den typischen neuronalen Hintergrund, das Neuropil, und eine geringe Anzahl (unter $5 \%$ ) an bereits teilweise differenzierten Neuroblasten. Die Kriterien einer teilweise differenzierten neuroblastischen Zelle sind ein mindestens doppelt so großer Zellkern mit Nukleolus und die doppelte Zytoplasmabreite, also eine synchrone Ausreifung von Kern und Zytoplasma in Richtung einer ausdifferenzierten Ganglienzelle im Vergleich zu einer unreifen neuroblastischen Zelle (• Abb. 1b). In differenzierenden Neuroblastomen liegt die Anzahl eben dieser 
Zellen über $5 \%$, der Hintergrund zeigt ausgedehnt Neuropil. Gerade im Randbereich oder in fibrovaskulären Septen können differenzierende Neuroblastome deutlich schwannzellreiches Stroma mit S100-Protein-positiven Schwannzellen aufweisen. Solange dieses jedoch unter $50 \%$ des Tumorvolumens ausmacht, stellt dies lediglich eine Differenzierung dar, die Kriterien eines Ganglioneuroblastoms sind jedoch nicht erfüllt.

\section{Gemischte Ganglioneuroblastome}

Gemischte Ganglioneuroblastome (- Abb. 2a) zeigen eine Mischung aus histomorphologischen Anteilen an Neuroblastomen und reifen Arealen mit Ganglienzellen in einem schwannzellreichen Stroma, dabei nehmen die ausdifferenzierten Anteile mehr als $50 \%$ ein. Die unreifen Neuroblasten liegen als kleine Herde untermischt (,intermixed") in den reifen Arealen. Seltener sind bereits makroskopisch ein oder multiple hämorrhagische Knoten abgrenzbar, die unreifen Neuroblastomanteilen entsprechen, diese Tumoren werden als noduläre Ganglioneuroblastome klassifiziert. Histologisch spiegelt sich das in einem abrupten Übergang von unreifen Anteilen zu einer schwannzellreichen differenzierten Komponente wider. Wenn eine Metastase eines Neuroblastoms z. B. im Knochenmark oder in Lymphknoten vorliegt, werden Ganglioneurome und -neuroblastome („intermixed“) automatisch als noduläre Ganglioneuroblastome entsprechend einer schlechteren klinischen Prognose klassifiziert. Eine weitere Variante nodulärer Ganglioneuroblastome zeigt makroskopisch lediglich einen großen Knoten, mit ausgedehnten Arealen eines Neuroblastoms und einem schmalen Rand entsprechend einem Ganglioneurom. Für die unreifen Anteile der nodulären Ganglioneuroblastome muss unbedingt auch der MKI und eventuell vorliegende Kalzifikationen angegeben werden, da diese Parameter in die Prognose eingehen.

\section{Klassisches noduläres Ganglioneuroblastom}

Im klassischen nodulären Ganglioneuroblastom ist die Neuroblastomkomponente entweder wenig differenziert oder dif-

Pathologe 2017-38:278-285 DOI 10.1007/s00292-017-0312-y

○ Der/die Autor(en) 2017. Dieser Artikel ist eine Open-Access-Publikation.

B. Gürtl-Lackner · D. Gisselsson-Nord · G. Vujanic

\section{Solide Kindertumoren. Ein Streifzug durch das Raritätenkabinett}

\section{Zusammenfassung}

Solide Kindertumoren sind extrem seltene Entitäten, die praktisch ausnahmslos in spezialisierten Zentren behandelt werden. Die Diagnose und weitere Therapie erfolgen gemäß gesamteuropäischen Studienprotokollen, daher werden die pathologische Aufarbeitung und Diagnosestellung anhand internationaler Leitlinien durchgeführt. Spezifische genetische Veränderungen spielen in der korrekten Diagnostik und für die nachfolgende Therapie beim Großteil der Tumoren eine wichtige Rolle, die entsprechenden Proben müssen daher im Rahmen der primären pathologischen
Beurteilung sichergestellt werden. Genetische Untersuchungen und eine referenzpathologische Zweitbegutachtung sind in den entsprechenden Leitlinien für alle soliden Kindertumoren vorgesehen. Neuroblastome, kongenitale mesoblastische Nephrome und Rhabdoidtumoren sind Beispiele für solide Tumoren, die nicht auf ein Organ beschränkt sind und nur im Kindesalter auftreten.

\section{Schlüsselwörter}

Neuroblastom $\cdot$ Kongenitales mesoblastisches Nephrom · Rhabdoidtumoren · MYC-N · INI1/SMARCB1

\section{Solid pediatric tumors. A brief survey of the rarity cabinet}

\section{Abstract}

Solid tumors in childhood are extremely rare entities, which are usually treated in specialized centers. Diagnosis and therapy are carried out according to a joint European protocol, whereby the pathological evaluation and therapy are carried out according to international guidelines. For the correct diagnosis and/or therapy of most tumors, analysis of specific genetic changes is mandatory; therefore, tumors have to be adequately sampled for parallel genetic analysis during the pathological work-up. A second opinion reference of the histopathological assessment is part of the international guidelines. Neuroblastomas, congenital mesoblastic nephromas and rhabdoid tumors are examples of solid tumors in childhood that are not restricted to one organ and occur exclusively during childhood.

\section{Keywords}

Neuroblastoma · Congenital mesoblastic nephroma $\cdot$ Rhabdoid tumors $\cdot$ MYC-N · INI1/SMARCB1 ferenzierend, der MKI gering oder mittel bei Kindern unter 1,5 Jahren, oder die Neuroblastomkomponente differenzierend mit einem geringen MKI bei Kindern zwischen 1,5 und 5 Jahren. Die Variante des klassischen nodulären Ganglioneuroblastoms umfasst jene Tumoren, die mit einer schlechteren Prognose assoziiert sind: Neuroblastomkomponenten mit hohem MKI oder undifferenzierte Neuroblastomkomponente bei allen Altersstufen, mittlerer MKI oder wenig differenzierte Neuroblastomkomponente bei Kindern älter als 1,5 Jahre und alle nodulären Ganglioneuroblastome bei Kindern über 5 Jahren (• Tab. 2).

Ganglioneurome enthalten schwannzellreiches Stroma und ausgereifte Ganglienzellen (• Abb. 2c), wobei der Begriff des reifenden Ganglioneuroms für Tu- moren angewendet wird, in welchen noch nicht vollständig ausgereifte Ganglienzellen identifiziert werden (• Abb. 2b; [21, 25, 26]).

\section{Genetische Parameter}

Das Neuroblastom war einer der ersten soliden Tumoren, bei dem bestimmte genetische Veränderungen identifiziert wurden, welche die Prognose und damit auch die Therapie des Patienten bestimmen. Die wichtigste genetische Veränderung ist eine Amplifikation (Vermehrung des genetischen Materials) des MYC-NGens aufChromosom 2p24 [24, 28]. Eine solche Amplifikation tritt bei ungefähr einem Viertel der Fälle auf und ist mit einer deutlich schlechteren Überlebensrate von weniger als $50 \%$ assoziiert [19]. 
Tab. 2 Beurteilungskriterien des klassischen nodulären Ganglioneuroblastoms

\begin{tabular}{|c|c|c|}
\hline Alter & Neuroblastomkomponente & MKI \\
\hline \multicolumn{3}{|c|}{ Klassisches noduläres Ganglioneuroblastom } \\
\hline \multirow[t]{2}{*}{$<1,5$ Jahre } & Wenig differenziert & Gering oder mittel \\
\hline & Differenzierend & Gering oder mittel \\
\hline Zwischen 1,5 und 5 Jahren & Differenzierend & Gering \\
\hline \multicolumn{3}{|c|}{ Variante des klassischen nodulären Ganglioneuroblastoms } \\
\hline Jedes Alter & Jede Differenzierung & Hoch \\
\hline Jedes Alter & Undifferenziert & Jeder \\
\hline$>1,5$ Jahre & Wenig differenziert & Jeder \\
\hline$>5$ Jahre & Jede Differenzierung & Hoch \\
\hline MKI Mitose-Karyorhexis-Inde & & \\
\hline
\end{tabular}

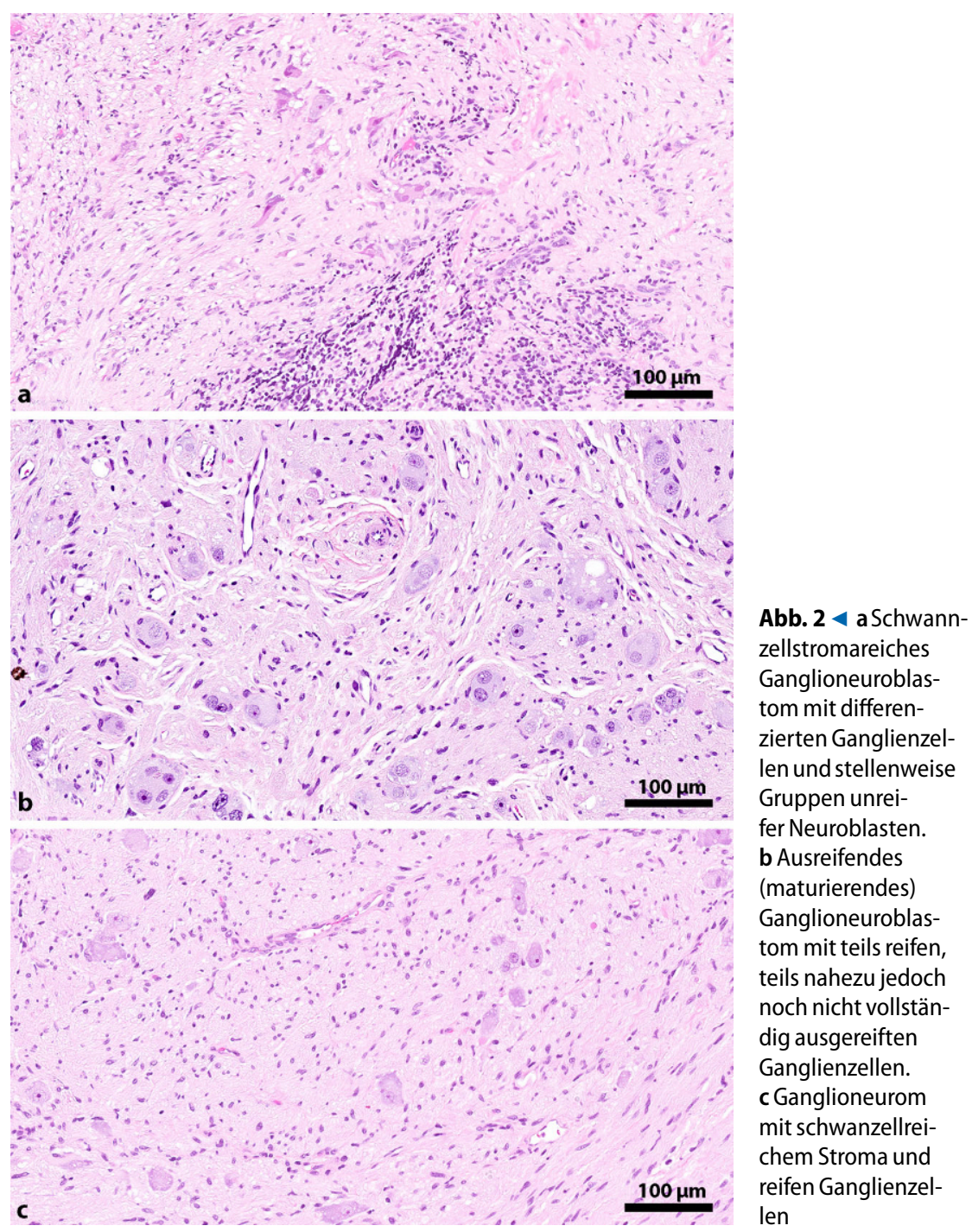

Relativ aktuelle Untersuchungen zeigen jedoch, dass jene Tumoren, die gut auf die chemotherapeutische Induktionstherapie ansprechen, auch im Falle einer MYC-N-Amplifikation eine vergleichbare Überlebensrate wie jene Tumoren ohne
MYC-N-Status als wichtigsten Parameter der Prognose aus [20]. Deletionen im langen Arm von Chromosom 11 sind mit einem geringeren Überlebenszeitraum assoziiert, unabhängig vom MYC-N-GenStatus. Tumoren mit dieser genetischen Veränderung sind typischerweise langsam wachsend und treten bei älteren Kindern auf [19]. Ein hyperdiploider und hypotetraploider DNA-Gehalt von Neuroblastomen ist mit einer guten Prognose assoziiert.

\section{Kongenitale mesoblastische Nephrome}

Kongenitale mesoblastische Nephrome repräsentieren ungefähr 2-5\% der Nierentumoren der frühen Kindheit. Die Mehrzahl manifestiert sich innerhalb der ersten 6 Monate oft auch kongenital [9]. Eine Manifestation nach dem 3. Lebensjahr ist extrem selten [4].

\section{Makroskopische Beurteilung}

Makroskopisch sind die Tumoren solide, mit einer glatten harten Oberfläche und einer leicht gelb gefärbten, faszikulären Schnittfläche [34] (•Infobox 2).

\section{Histologie und histologische Subtypen}

Histologisch werden 3 Subtypen unterschieden, der klassische Typ (• Abb. 3a), welcher ungefähr $24 \%$ der Fälle ausmacht, und der zelluläre Typ (• Abb. 3b), welcher in ungefähr $66 \%$ der Fälle vorliegt. In bis zu $10 \%$ der Fälle liegen Areale beider Subtypen vor. Entsprechend der gängigen Definition wird die Unterscheidung zwischen klassischen und zellulären mesoblastischen Nephromen anhand der Zellularität getroffen [12].

Klassische kongenitale mesoblastische Nephrome zeigen fibroblastenähnliche Zellen in Faszikeln und eine niedrige mitotische Aktivität. Zelluläre kongenitale mesoblastische Nephrome hingegen weisen einen höheren Grad an Zellulärität auf, schlechter ausgeformte Faszikel, eine höhere mitotische Aktivität und Nekroseareale. In der zellulären Variante wurden auch Nukleolen beschrieben [4]. Mesoblastische Nephrome haben 


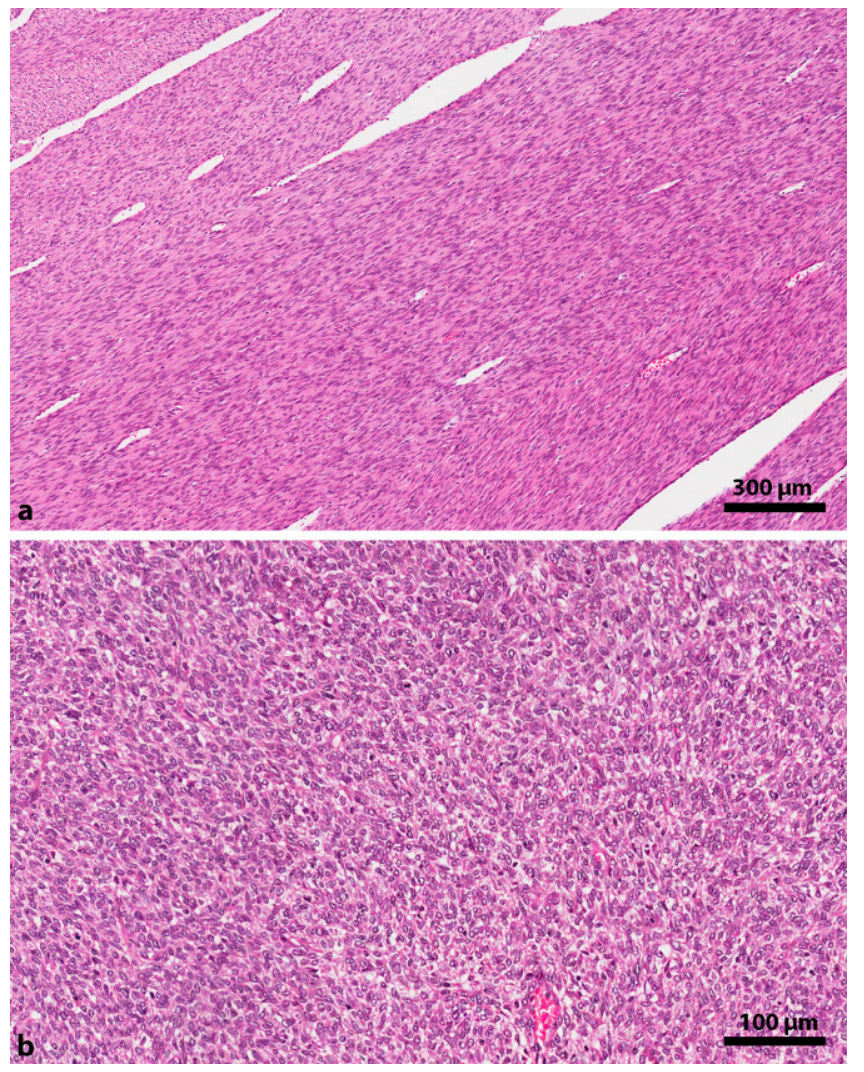

Abb. $3<$ a Klassisches kongenitales mesoblastisches Nephrom mit infiltrativem Wachstum in das Nierenparenchym.bZelluläre Variante mit hoher Zellularität und deutlicher mitotischer Aktivität insbesondere im Vergleich zua kein charakteristisches immunhistochemisches Muster, können Positivität für SMA, Desmin, Vimentin und Zytokeratin aufweisen, in Einzelfällen aber auch für andere Marker [12].

\section{Genetische Parameter}

Ein wichtiger diagnostischer Durchbruch war die Entdeckung der typischen Translokation zwischen dem ETV6und dem NTRK-3-Gen. Die In-situ-Hybridisierung klassischer und zellulärer mesoblastischer Nephrome und infantiler Fibrosarkome zeigte das Vorliegen der oben beschriebenen Translokation in zellulären mesoblastischen Nephromen und infantilen Fibrosarkomen [3, 16].

Zelluläre mesoblastische Nephrome zeigen damit nicht nur eine identische Histologie und Ultrastruktur wie infantile Fibrosarkome [22], sondern zudem die gleiche Translokation wie infantile Fibrosarkome, welche zur Expression des ETV6-NTRK3-Transkripts führt $[4,22]$. Die zelluläre Variante des mesoblastischen Nephroms scheint somit eine in der Niere lokalisierte Variante des infantilen Fibrosarkoms mit der identischen genomischen Translokation $\mathrm{t}(12 ; 15)(\mathrm{p} 13 ; \mathrm{q} 25) \mathrm{zu}$ repräsentieren [5].

Für den Großteil der Patienten mit kongenitalem mesoblastischem $\mathrm{Ne}$ phrom ist die Prognose unabhängig vom Subtyp ausgezeichnet. Derzeit werden beide Subtypen chirurgisch behandelt, wobei die Resektionsrandanalyse des Pathologen einen enorm wichtigen Indikator für die weitere Prognose des Patienten darstellt $[9,11]$.

In den meisten Fällen ist die chirurgische Exzision kurativ; insbesondere für die zelluläre Variante sind jedoch Fälle mit Metastasierung beschrieben [31].

\section{Rhabdoidtumor}

Der maligne Rhabdoidtumor tritt typischerweise im Kleinkindalter auf; $90 \%$ der Tumoren werden innerhalb der ersten 3 Lebensjahre diagnostiziert. Ursprünglich wurde der Rhabdoidtumor als Nierentumor beschrieben, in der Folge aber auch in anderen Körperregionen wie beispielsweise den Weichteilen, der Leber oder den Gallenwegen. Im ZNS gibt es das atypische Teratom/ Rhabdoidtumor mit identischer Mor-

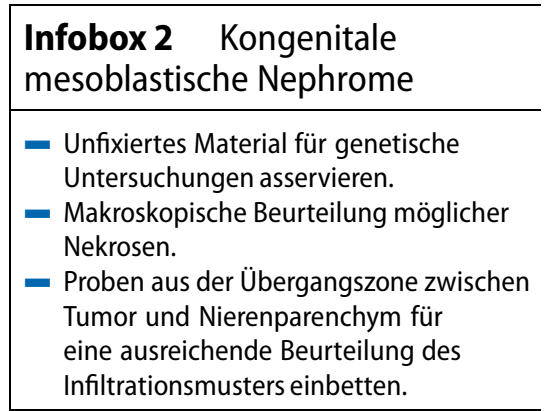

phologie und immunhistochemischer Charakteristik. Klinisch präsentiert sich der Tumor oftmals in einem fortgeschrittenen Stadium, teils bereits mit intrakranieller Metastasierung, und hat insgesamt eine schlechte Prognose [32, 33].

\section{Makroskopische Beurteilung}

Makroskopisch stellen sich Rhabdoidtumoren grauweiß, leicht zerreißlich mit Nekrosearealen und Einblutung dar. Das Tumorgewebe ersetzt gewissermaßen das Nierenparenchym, und oft ist nur mehr ein schmaler Saum an normalem Nierengewebe vorhanden [33].

\section{Histologie und histologische Subtypen}

Histologisch zeigen Rhabdoidtumoren ein solides Proliferat an Zellen. Die für den Tumor charakteristischen Zellen sind zytoplasmareich, mit typischen intrazytoplasmatischen Inklusionen (• Abb. 4b; [27]). Die Zellkerne sind oft exzentrisch, groß, mit einem deutlichen Nukleolus. Die intrazytoplasmatischen Inklusionen stellen sich elektronenmikroskopisch als Ansammlung von Intermediärfilamenten dar und sind immunhistochemisch positiv für Vimentin, teilweise Zytokeratin, EMA sowie Desmin [32, 33]. Neben diesem klassischen Wachstumsmuster gibt es morphologisch ein weites Spektrum, welches beispielsweise das sklerosierende, epitheloide, spindelzellige, lymphozytenreiche oder gefäßreiche Wachstumsmuster umfasst. Die Tumoren zeichnen sich auch histologisch durch ein sehr aggressives Wachstum mit Zeichen der Kapselinvasion, Gefäß- 


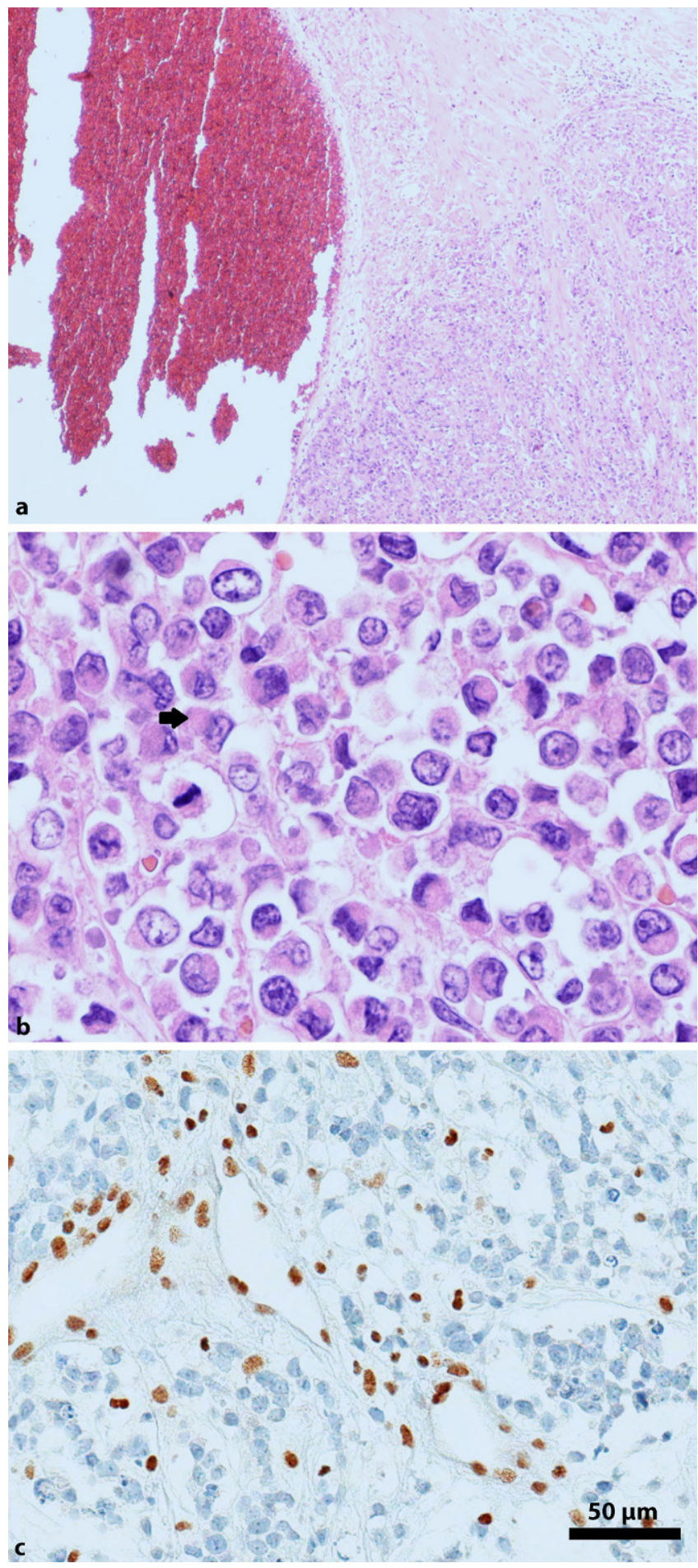

Abb. $4<$ a Infiltration eines $\mathrm{Ge}$ fäßes des Nierenhilus durch einen Rhaboidtumor. b Typische Morphologie eines solide wachsenden Rhabdoidtumors: die Tumorzellen zeigen große eosinophile zytoplasmatische Inklusionen. c Immunhistochemische Untersuchung mit Antikörper gegen INI1/ SMARCB1. Endothelzellen mit erhaltener Kernfärbung, vollständige Negativität der Tumorzellen

einbrüchen ( $\bullet$ Abb. 4a) und Einbruch ins Nierenbecken aus [33].

Gerade extrarenal liegen oft ausgedehnte Nekrosen und Einblutungen vor, sodass die typischen Zellen extrem schwer zu identifizieren sind. Teilweise präsentieren sich diese Tumoren auch morphologisch als klein-, rund- und blauzelliger Tumor; dies erschwert die

\begin{tabular}{|l}
\hline Infobox $3 \quad$ Rhabdoidtumor \\
\hline - Unfixiertes Material für genetische \\
Untersuchungen asservieren. \\
- Histologische Proben stets auch aus \\
Randarealen von Nekrosen entnehmen \\
zur Identifikation typischer rhabdoider \\
Zellen. \\
- Differenzialdiagnostisch schwierige \\
kleinzellige Tumoren zum Ausschluss eines \\
Rhabdoidtumors mit Antikörper gegen \\
SMARCB1 und SMARCA4 gegentesten.
\end{tabular}

derungen des Tumorsuppressor-Gens INI1/SMARCB1 (hSNF5-Gens) als relativ spezifische und konstante genetische Veränderung. Dabei kommt es aufgrund kombinierter genetischer Veränderungen zu einem Ausfall beider Allele [6,30]. INI1 ist Teil eines Proteinkomplexes, der Veränderungen der Chromatinstruktur im Rahmen der Transkription von Genen bewirkt [7]. Eine kleine Anzahl an Rhabdoidtumoren zeigt allerdings genetische Veränderungen des Tumorsuppressor-Gens SMARCA4, welches dem gleichen Proteinkomplex angehört [23]. Bei Vorliegen der beschriebenen genetischen Veränderungen kommt es $\mathrm{zu}$ einem Ausfall der Proteinexpression, welcher für beide TumorsuppressorGene sehr gut immunhistochemisch nachgewiesen werden kann. Dabei sind die Tumorzellen vollständig negativ, alle anderen Zellen wie beispielsweise Endothelzellen oder Fibroblasten nukleär deutlich positiv ( $\bullet$ Abb. 4c; $\bullet$ Infobox 3; [14]). Differenzialdiagnostisch sollte allerdings berücksichtigt werden, dass die oben beschriebenen genetischen Veränderungen nicht vollständig spezifisch sind, sondern auch in anderen Tumorentitäten wie beispielsweise dem epitheloiden Sarkom oder kleinzelligen Ovarialkarzinom vorliegen können [2, 10].

\section{Fazit für die Praxis}

- Nahezu alle soliden Tumoren des Kindesalters werden von klinischer, pathologischer und genetischer Seite in internationale Studien eingebracht.

Untersuchungen 13 verschiedener Zelllinien von Rhabdoidtumoren und die parallele Analyse der Primärtumoren charakterisierten chromosomale Verän-
- Material solider Kindertumoren ist für entsprechende genetische Studien 


\section{gemäß den vorliegenden Richtlinien zu sichern. \\ - Bei der Beurteilung muss immer die klinische Korrelation mit in Be- tracht gezogen werden. Dafür ist das Gespräch mit den klinischen behan- delnden Kollegen (Kinderchirurgie, Kinderonkologie) zu suchen.}

\begin{tabular}{l} 
Korrespondenzadresse \\
\hline $\begin{array}{l}\text { Assoz. Prof. Dr. B. Gürtl- } \\
\text { Lackner } \\
\text { Institut für Pathologie, } \\
\text { Labmedicin Skåne } \\
\text { Sölvegatan 25, Lund, } \\
\text { Schweden } \\
\text { Barbara.GurtlLackner@ } \\
\text { skane.se }\end{array}$ \\
\hline
\end{tabular}

Danksagung. Der Artikel ist gewidmet Professor Ivo Leuscher, verstorben am 29.01.2017, dessen spezielles berufliches Interesse Tumoren im Kindesalter galt und der uns als hervorragender Diagnostiker und hilfsbereiter Kollege immer in Erinnerung bleiben wird.

Open access funding provided by Medical University of Graz.

\section{Einhaltung ethischer Richtlinien}

Interessenkonflikt. B. Gürtl-Lackner, D. GisselssonNord und G. Vujanic geben an, dass kein Interessenkonflikt besteht.

Dieser Beitrag beinhaltet keine von den Autoren durchgeführten Studien an Menschen oder Tieren.

Open Access. Dieser Artikel wird unter der Creative Commons Namensnennung 4.0 International Lizenz (http://creativecommons.org/licenses/by/4.0/deed. de) veröffentlicht, welche die Nutzung, Vervielfältigung, Bearbeitung, Verbreitung und Wiedergabe in jeglichem Medium und Format erlaubt, sofern Sie den/die ursprünglichen Autor(en) und die Quelle ordnungsgemäßnennen, einen Link zur Creative Commons Lizenz beifügen und angeben, ob Änderungen vorgenommen wurden.

\section{Literatur}

1. Ambros PF, Ambros IM, Brodeur GM, Haber M, Khan J, Nakagawara A, Schleiermacher G, Speleman F, Spitz R, London WB, Cohn SL, Pearson $A D$, Maris JM (2009) International consensus for neuroblastoma molecular diagnostics: report from the International Neuroblastoma Risk Group (INRG) Biology Committee. Br J Cancer 100:1471-1482

2. Agaimy A (2014) The expanding family of SMARCB1(INI1)-deficient neoplasia: implications of phenotypic, biological, and molecular heterogeneity. Adv Anat Pathol 21:394-410
3. Argani P, Fritsch M, Kadkol SS, Schuster A, Beckwith JB, Perlman EJ (2000) Detection of the ETV6-NTRK3 chimeric RNA of infantile fibrosarcoma/cellular congenital mesoblastic nephroma in paraffinembedded tissue: application to challenging pediatric renal stromal tumors. Mod Pathol 13:29-36

4. Argani P, Ladanyi M (2003) Recent advances in pediatric renal neoplasia. Adv Anat Pathol 10:243-260

5. Argani P, Sorensen PHB (2004) Congenital mesoblastic nephroma. In: Eble JN, Sauter G, Epstein Jl, Sesterhenn IA (Hrsg) Pathology \& genetics. Tumours of the urinary system and male genital organs. World Health Organization Classification of Tumours. International Agency for Research on Cancer Press, Lyon, S60-61

6. Biegel JA, Zhou JY, Rorke LB, Stenstrom C, Wainwright LM, Fogelgren B (1999) Germ-line and acquired mutations of INI 1 in atypical teratoid and rhabdoid tumors. Cancer Res 59:74-79

7. Biegel JA, Busse TM, Weissman BE (2014) SWI/SNF chromatin remodeling complexes and cancer. Am JMed Genet C Semin Med Genet 166C:350-366

8. BurchillSA, BeiskeK, Shimada H, Ambros PF, Seeger R, Tytgat GAM, Brock PR, Haber M, Park JR, Berthold $F(2016)$ Recommendations for the standardization of bone marrow disease assessment and reporting in children with neuroblastoma; on behalf of the International Neuroblastoma Response Criteria Bone Marrow Working Group. Cancer. doi:10.1002/ cncr.30380

9. England RJ, Haider N, Vujanic GM, Kelsey A, Stiller CA, Pritchard-Jones K, Powis M (2011) Mesoblastic nephroma: A report of the United Kingdom Children's Cancer and Leukemia Group (CCLG). Pediatr Blood Cancer 56:744-748

10. Fuller CE (2016) All things rhabdoid and SMARC: An enigmatic exploration with Dr. Louis P. Dehner. Semin Diagn Pathol. doi:10.1053/j.semdp.2016. 08.003

11. Furtwängler $R$, Reinhard $H$, Leuschner I, Schenk JP, Goebel U, Claviez A, Kulozik A, Zoubek $A$, von Schweinitz D, Graf N, Gesellschaft für Pädiatrische Onkologie und Hämatologie (GPOH) Nephroblastoma Study Group (2006) Mesoblastic nephroma - a report from the Gesellschaft für Pädiatrische Onkologie und Hämatologie (GPOH). Cancer 106:2275-2283

12. Gooskens SL, Houwing MA, Vujanic GM, Dome JS, Diertens T, Coulomb-I'Herminé A, Godzinski J, Pritchard-Jones K, Graf N, vanden Heuvel-Eibrink MM (2017) Congenital mesoblastic nephroma 50 years after its recognition: a narrative review. Pediatr Blood Cancer 00:e26437

13. Gururangan $S$, Bowman LC, Parham DM, Wilimas JA, Rao B, Pratt CB, Douglass EC (1993) Primary extracranial rhabdoid tumors. Clinicopathologic features and response to ifosfamide. Cancer 71:2653-2659

14. Hasselblatt M, Gesk S, Oyen F, Rossi S, Viscardi E, Giangaspero F, Giannini C, Judkins AR, Frühwald MC, Obser T, Schneppenheim R, Siebert R, Paulus W (2011) Nonsense mutation and inactivation of SMARCA4 (BRG1) in an atypical teratoid/ rhabdoid tumor showing retained SMARCB1 (INI1) expression. Am J Surg Pathol 35:933-935

15. Huang M, Weiss WA (2013) Neuroblastoma and MYCN. Cold Spring Harb Perspect Med 3:a014415. doi:10.1101/cshperspect.a014415

16. Knezevich SR, Garnett MJ, Pysher TJ, Beckwith JB, Grundy PE, Sorensen PH (1998) Histogenetic link between mesoblastic nephroma and congenital fibrosarcoma. Cancer Res 58:5046-5048
17. Kushner BH, Modak S, Kramer K, LaQuaglia MP Yataghene K, Basu EM, Roberts SS, Kushner BH, Modak S, Kramer K, LaQuaglia MP, Yataghene K, Basu EM, Roberts SS, Cheung NK (2014) Striking dichotomy in outcome of MYCN-amplified neuroblastoma in the contemporary era. Cancer 120:2050-2059

18. Madigan CE, Armenian SH, Malogolowkin MH, Mascarenhas L (2007) Extracranial malignant rhabdoid tumors in childhood: the Childrens Hospital Los Angeles experience. Cancer 110:2061-2066

19. Maris JM, Hogarty MD, Bagatell R, Cohn SL (2007) Neuroblastoma. Lancet 369:2106-2120

20. Nakazawa A, Haga C, Ohira M, Okita H, Kamijo T, Nakagawara A (2015) Correlation between the International Neuroblastoma Pathology Classification and genomic signature in neuroblastoma. Cancer Sci 106:766-771

21. Peuchmaur $M$, d'Amore ES, Joshi VV, Hata J, Roald B, Dehner LP, Gerbing RB, Stram DO, Lukens JN, Matthay KK, Shimada H (2003) Revision of the International Neuroblastoma Pathology Classification: confirmation of favorable and unfavorable prognostic subsets in ganglioneuroblastoma, nodular. Cancer 98:2274-2281

22. Rubin BP, Chen Cj MTW, Xiao S, Grier HE, Kozakewich HP, Perez-Atazde AR, Fletcher JA (1998) Congenital mesoblastic nephroma t(12;15) is associated with ETV6-NTRK3 gene fusion. Am J Pathol 153:1451-1458

23. Schneppenheim R, Frühwald MC, Gesk S, Hasselblatt M, Jeibmann A, Kordes U, Kreuz M, Leuschner I, Subero MJ, Obser T, Oyen F, Vater I, Siebert R (2010) Germline nonsense mutation and somatic inactivation of SMARCA4/BRG1 in a family with rhabdoid tumor predisposition syndrome. Am J Hum Genet 86:279-284

24. Schwab M, Alitalo K, Klempnauer KH, Varmus HE, Bishop JM, Gilbert F, Brodeur G, Goldstein M, Trent J (1983) Amplified DNA with limited homology to myc cellular oncogene is shared by human neuroblastoma cell lines and a neuroblastoma tumour. Nature 305:245-248

25. Shimada H, Ambros IM, Dehner LP, Hata J, Joshi VV, Roald B (1999) Terminology and morphologic criteria of neuroblastic tumors. Cancer 86:349-363

26. Shimada $H$, Umehara $S$, Monobe $Y$, Hachitanda Y, Nakagawa A, Goto S, Gerbing RB, Stram DO, Lukens JN, Matthay KK (2001) International neuroblastoma pathology classification for prognostic evaluation of patients with peripheral neuroblastic tumors. Cancer 92:2451-2461

27. Sotelo-Avila C, Gonzalez-Crussi F, deMello D, Vogler C, Gooch WM 3rd, Gale G, Pena R (1986) Renal and extrarenal rhabdoid tumors in children: a clinicopathologic study of 14 patients. Semin Diagn Pathol 3:151-163

28. Spitz R, Hero B, Skowron M, Ernestus K, Berthold F (2004) MYCN-status in neuroblastoma: characteristics of tumours showing amplification gain and non-amplification. Eur J Cancer 40:2753-2759

29. Suganuma R, Wang LL, Sano H, Naranjo A, London WB, Seeger RC, Hogarty MD, Gastier-Foster JM, Look AT, Park JR, Maris JM, Cohn SL, Amann G, Beiske K, Cullinane CJ, d'Amore ES, Gambini C, Jarzembowski JA, Joshi VV, Navarro S, Peuchmaur M, Shimada H (2013) Peripheral neuroblastic tumors with genotype-phenotype discordance: a report from the Children's Oncology Group and the International Neuroblastoma Pathology Committee. Pediatr Blood Cancer 60:363-370

30. Versteege I, Sévenet N, Lange J, Rousseau-Merck MF, Ambros P, Handgretinger R, Aurias A, Delattre 
O (1998) Truncating mutations of hSNF5/INI1 in aggressive paediatric cancer. Nature 394:203-206

31. Vujanic GM, Delamarre JF, Moeslichan S, Lam J, Harms D, Sandstedt B, Voûte PA (1993) Mesoblastic nephroma metastatic to the lungs and heart Anotherface of this peculiarlesion: Case reportand review of the literature. Pediatr Pathol 13:143-153

32. Vujanic GM, Sandstedt B, Harms D, BocconGibod L, Delemarre JFM (1996) Rhabdoid tumors of the kidney: a clinicopathological study of 22 patients from the International society of Paediatric Oncology (SIOP) nephroblastoma file. Histopathology 28:333-340

33. Weeks DA, Beckwith JB, Mierau GW, Luckey DW (1989) Rhabdoid tumor of kidney. A report of 111 cases from the National Wilms' Tumor Study Pathology Center. Am J Surg Pathol 13:439-458

34. Young RH, Eble JN (2002) Tumors of the urinary tract. In: Fletcher CD (Hrsg) Diagnostic histopathology of tumors, 2. Aufl. Elsevier Science, Philadelphia, S559-602

\section{Krenn, G. Perino \\ Histological Diagnosis \\ of Implant-Associated \\ Pathologies}

Clinical Management of Joint

Arthroplasty

Berlin/Heidelberg: Springer 2017,

1. Auflage, 44 S., (ISBN: 978-3-662-

54204-0), 18,99 EUR

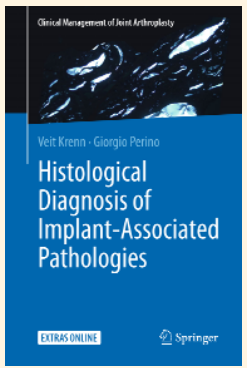

Das vorliegende Buch (43 Seiten, 68 Literaturzitate) bietet einen umfassenden jedoch zugleich fokussierten Einblick in den sich schnell wandelnden Bereich der Gelenkimplantat-

assoziierten-Pathologie.

Die Gelenkendoprothetik zählt zu den erfolgreichsten Eingriffen der operativen Orthopädie und Traumatologie und führt nachweislich zu deutlicher Schmerzreduktion, Gewinn an Mobilität und somit verbesserter Lebensqualität. Trotzdem bestehen zu einem Prozentsatz von etwa 5-10\% Pathologien, die eine große diagnostische und therapeutische Herausforderung für den Patienten und die Orthopädie/Unfallchirurgie darstellen. Die histopathologische Diagnostik ist ein integraler Bestandteil in der Abklärung eines Gelenkendoprothesen-Versagens und trägt zur Ursachenabklärung von entzündlichen Prozessen, infektiösen Prozessen und fremdkörperinduzierten Reaktionen bei. Mit dem vorliegenden Buch ist es gelungen die mittlerweile international akzeptierte Konsensusklassifikation der Gelenkendoprothetik und den damit verbundenen Algorithmus zur Identifikation von Gelenkprothesenmaterialien, in einer übersichtlichen Form zu veranschaulichen. Auf Seite 4 befindet sich das Herzstück dieser Klassifikation (SLIM Konsensusklassifikation), welche international publiziert wurde und auf einer Zusammenarbeit aus 6 unterschiedlichen Ländern basiert.

Der Anspruch dieser histopathologischen Klassifikation ist die Gesamtheit an Endoprothesen-assoizierten Pathologien zu diagnostizieren: die TYP I-Membranen, partikelinduziert; die Typ II-Membranen, infektiösinduziert; die Typ III-Membran, eine Kom- bination aus Abriebreaktion und Infektion; und die Typ VI-Membran, die sogenannte indifferente Membran als äquivalent von funktionellen Ursachen. Diese Membrantypen stellen die Grundlage für die erweiterte Diagnostik von Arthrofibrose, inflammatorischer Reaktion wie bei dysfunktionalen Prothesen mit Metall-Metallgleitpaarung und Implantatmaterialallergien dar. Ergänzt und abgerundet wird das Spektrum durch die periimplantäre Knochenpathologie, welche die aseptische Knochennekrose und Osteopenie umfasst. Die Charakterisierung der Abriebpartikel ist ein weiterer wesentlicher Bestandteil des diagnostischen Algorithmus. Insbesondere ist hier die Abgrenzung von Prothesenmaterial und Nicht-Prothesenmaterial (beispielsweise Urat oder Kalziumphosphat) entscheidend.

Hervorzuheben ist in diesem Buch außerdem die genaue mikroskopische Charakterisierung einer großen Anzahl von Prothesenpartikel. Das Buch bietet darüber hinaus nützliche Beschreibungen zur standardisierten Entnahme von Gewebeproben.

In der Zusammenfassung wird darauf verwiesen, dass die Abklärung dieser komplexen Pathologien letztendlich immer in einem interdisziplinären Kontext zu erfolgen hat. Eine umfassende Diagnostik ist nur durch die Zusammenschau von Befunden der Orthopädie/Unfallchirurgie, der bildgebenden Verfahren, der Mikrobiologie, der Biomechanik und der histopathologische Diagnostik möglich.

Die Zielgruppe besteht in Kolleginnen und Kollegen aus der Orthopädie/Unfallchirurgie mit Schwerpunkt Gelenkendoprothetik, der Mikrobiologie, der Radiologie und natürlich der Pathologie.

Das Buch zeichnet sich durch die gut handhabbare Größe und die bewusst knappen Darstellungen aus. Der systematische Aufbau, die Erklärung sämtlicher Abkürzungen und die farbige Illustration der teils ästhetisch wirkenden histopathologischen Präparate runden das Erscheinungsbild ab.

Das vorliegende Buch bietet die Grundlage für eine standardisierte und auf Basis von zwei Algorithmen festgelegte, interdisziplinäre Diagnostik mit besonderer Berücksichtigung der histopathologischen Diagnose.

W. Waldstein (Wien, Österreich) 